\title{
Numerical Simulation of Fluid Flow Features of Mechanical Mixer with Automatic Variable Frequency Control
}

\author{
Bing Yang \\ Zibo Vocational Institute, Zibo 255314, China
}

Corresponding Author Email: 10429@zbvc.edu.cn

https://doi.org/10.18280/ijht.390135

Received: 16 October 2020

Accepted: 29 December 2020

\section{Keywords: \\ variable frequency control, mechanical mixer, fluid flow features, numerical simulation}

\begin{abstract}
For many engineering fields, it is of practical significance to construct and reasonably simplify the solid-liquid two-phase flow model in the cylinder of mechanical mixer. However, the existing studies have not considered how the control technology affects the fluid features in the mixer. From the angle of automatic variable frequency control, this paper carries out a numerical simulation of the fluid flow features in mechanical mixer. Firstly, the authors constructed the system structure for the mixer with automatic variable frequency control, and summarized the basic devices and the number of input/output points of the system. Then, the relationship between the force energy and control frequency of the mixing cylinder was detailed. Afterwards, the Euler-Euler two fluid model was introduced to numerically simulate the solid-liquid two-phase flow in the cylinder of the mixer, which contains both solid particles and liquid, followed by the establishment of control equation, the drag model, and the turbulence model. The proposed model was proved effective through experiments. The research findings provide a reference for the relevant research and the optimal design of the drive module of mixers.
\end{abstract}

\section{INTRODUCTION}

In metallurgy, mining, and biology, a common reactor is the mixer capable of mixing various raw materials. To capture the mixing properties and optimize the structure of the mixer, it is necessary to deeply examine the mixing and flow features of the solid-liquid two phase in the mixing cylinder [1-4]. The existing measuring devices for flow field features are troubled by three defects: the devices are too small, the measuring error is high due to the empirical formula, and the detail features are not described clearly [5-8]. For many engineering fields, it is of practical significance to construct and reasonably simplify the solid-liquid two-phase flow model in the cylinder of mechanical mixer. The relevant findings could provide a reference for the relevant research and the optimal design of the drive module of mixers.

In material processing and molding, the mixing, transport, and self-cleaning of solid and liquid materials can be realized through the continuous operation of mixing and kneading machines [9-13]. Panneerselvam et al. [14] summarized the structural features and working mechanism of differential mixer and kneader, established a mathematical model of the system and the tooth equation for the end surface of the mixing shaft, and conducted a systematic Fluent analysis on the flow field features of the materials in the mixing cylinder under different stresses, strains, and viscosities. Focusing on the mixing and settling cylinder, Meridiano et al. [15] constructed the turbulence model and multi-phase flow model of the system, carried out numerical simulation of the broken and clustering of droplets, and explored the impact of solid-liquid phase dispersion and flow field distribution under changing system structure and operating parameters; Comparing the practicality of population balance model with that of damage model, and they acquired the flow field distribution of the liquid phase, and the dispersion phase in the mixing cylinder, under different paddles.

In recent years, the popular microfluidic technology has been extensively applied to biochemical fields, by virtue of its fast analysis speed of the reagents [16-18]. Targeting the difficult laminar mixing problem with low Reynolds number, Ricci and Kelly [19] analyzed the fluid mixing features in micro-scale, designed two microscale rapid mixers, namely, the electric field-driven electroosmosis micromixer and the passive micromixer with a contracted lateral flow wall, and evaluated the mixing efficiency and performance of the two mixers through experiments. Zughbi and Raki [20] introduced the large triangular paddle, known for its excellent mixing performance, to the multistage mixing and settling cylinder, and optimized the structure of large triangular paddle and mixing system; finally, the optimized paddle was proved as effective in improving the mixing performance and suction ability of the system, and feasible in applying to largescale industrial mixing and settling cylinder.

Zughbi and Rakib [21] analyzed the fluid mechanics of mechanical mixers that are widely used in food, oil, and biological industries, and systematically analyzed the global or local flow field information, such as dissipation rate of turbulent energy, shear rate, and velocity, through threedimensional (3D) geometric model, further revealing the flow behavior of the fluid. After improving the power number association of mixer power, $\mathrm{Wu}$ et al. [22] constructed the relationship formulas for the shaft distance between stator and rotor, and the radial distance from the rotor to the mixing cylinder wall based on the prediction of system power consumption, and realized accurate forecast of bubble size and distribution. 
Most of today's mixers are controlled by configuration software, programmable logical controller (PLC), and variable frequency velocity control. However, there is not yet any literature about how these control techniques affect the fluid features in the mixing cylinder of the mechanical mixer. To solve the problem, this paper carries out numerical simulation of fluid flow features in mechanical mixer, from the perspective of automatic variable frequency control. Firstly, the authors set up the structure of the application system for the mixer with automatic variable frequency control, and summarized the basic devices and the number of input/output points of the system. Next, the relationship between the force energy of the mixing cylinder and control frequency was detailed. After that, the Euler-Euler two fluid model was introduced to numerically simulate the solid-liquid two-phase flow in the cylinder of the mixer, which contains both solid particles and liquid, followed by the establishment of control equation, the drag model, and the turbulence model. Finally, experiments were carried out to verify the effectiveness of our model, and the fluid flow features were analyzed under different volume fractions of the particles.

\section{STRUCTURE OF MECHANICAL MIXER WITH AUTOMATIC VARIABLE FREQUENCY CONTROL}

As shown in Figure 1, the above system consists of 7 solenoid valves that control the feeding of each stock bin, 7 photoelectric sensors to measure the materials in the stock bins, 3 transmission motors, 2 mixing motors, 5 velocity sensors to capture the motor velocity, 5 inverters for driving motors, and 7 relays.

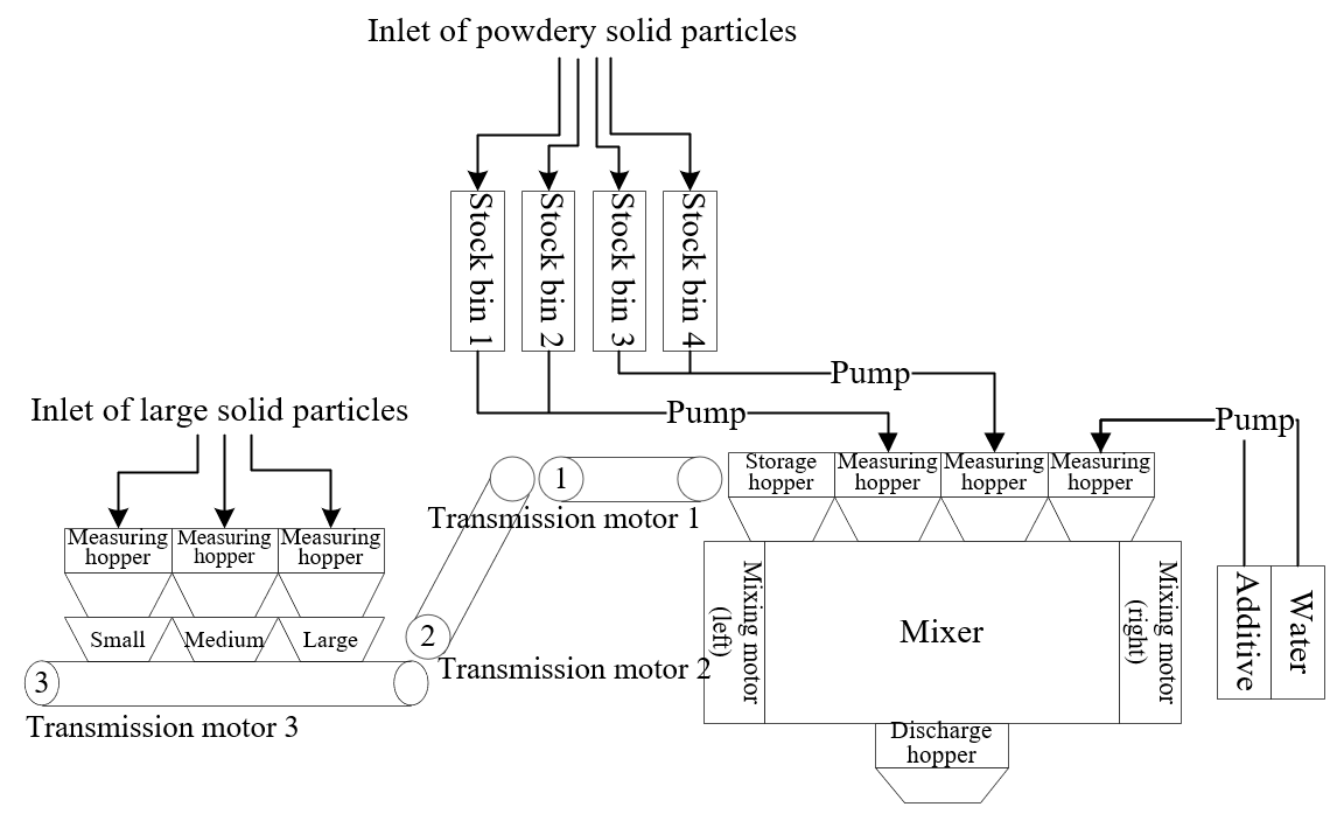

Figure 1. Structure of application system for the mixer with automatic variable frequency control

Table 1. Number of input/output points

\begin{tabular}{ccccccc}
\hline Type & Analog output & Analog input & & \multicolumn{2}{c}{ Switched output } & Switched input \\
\hline Type of signal & Frequency & Velocity & Motor on/off? & Mixer on/off? & Solenoid valve on/off? & Feed yes/no? \\
Number & 5 & 5 & 5 & 3 & 7 & 7 \\
Total & 5 & 5 & & 29 & 12 & 7 \\
Grand total & & & & 29 & & \\
\hline
\end{tabular}

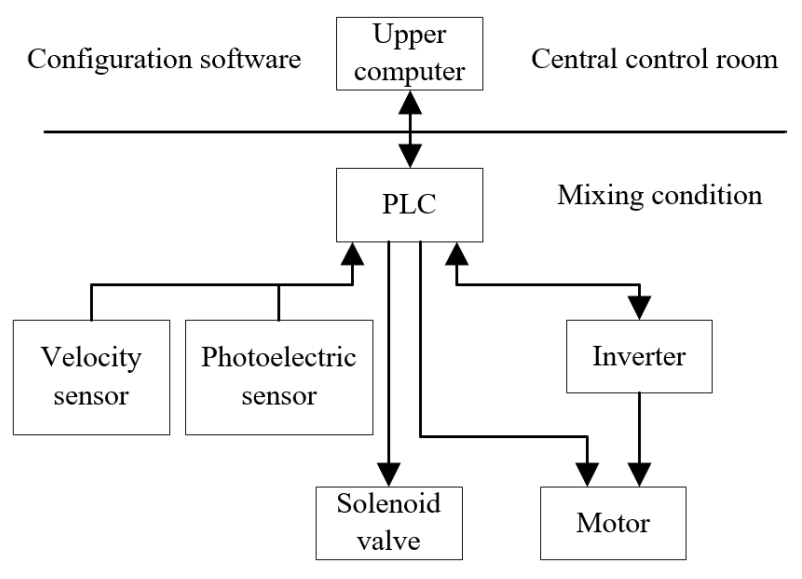

Figure 2. Mixer control system
Table 1 shows the number of input/output points of the system. A total of 29 input/output points were taken as the controlled objects. The motor velocity was jointly regulated by the PLC and inverter. The output current of the PLC serves as the controlled input to the inverter. Then, the inverter modulates and outputs a controllable frequency signal to the asynchronous motor. Meanwhile, the PLC is controlled by the upper computer. The control system is illustrated in Figure 2.

\section{RELATIONSHIP BETWEEN FORCE ENERGY AND CONTROL FREQUENCY}

Let $S E$ be the mixing energy of the mixing cylinder, $Q_{M}$ be the mass of the fluid in the cylinder, $I N, L S$, and $\theta$ be the rotational inertia, linear velocity, and angular velocity of the mixing paddle, respectively, and $g$ be the lead of paddle shaft. 
Under the action of the drive motor, the energy stored in the moving part of the mixer during the mixing can be expressed as:

$$
S E=\frac{1}{2} Q_{M} L S^{2}+\frac{1}{2} I N \theta^{2}=\frac{1}{2}\left(\frac{g^{2}}{4 \pi^{2}} Q_{M}+I N\right) \theta^{2}
$$

Let $r$ be the transmission ratio, and $\theta_{M O}$ be the angular velocity of the motor. When the motor is directly driven by the inverter, there exists $\theta=\theta_{M O} / r$. Thus, we have:

$$
S E=\frac{1}{2}\left(\frac{g^{2}}{4 \pi^{2}} Q_{M}+I N\right) \theta^{2}=\frac{1}{2} I N_{M O} \theta_{M O}^{2}
$$

The equivalent rotational inertia $I N_{M O}$ of the drive motor shaft can be expressed as:

$$
\left(\frac{g^{2}}{4 \pi^{2}} Q_{M}+I N_{M O}\right) \frac{1}{r^{2}}
$$

The stressed parts of the mixer include the fuselage, paddle, paddle shaft, etc. When the mixing becomes stable, the paddle velocity also tends to be stable. The mixing energy will be converted into the work $W_{W D}$ that promotes the liquid to flow in the mixing cylinder, the elastic deformation work $W_{E D}$ of the stressed parts, and the friction work $W_{F R}$ consumed by the fluid flow to overcome the frictions brought by the stressed parts or the mixing cylinder:

$$
S E=W_{W D}+W_{E D}+W_{F R}
$$

Let $F O_{W D}$ be the force exerted to promote the flow of the liquid in the cylinder, and $\varepsilon$ be the unit volume of the liquid in the cylinder. Then, the $W_{W D}$ that varies with processing techniques can be calculated by:

$$
W_{W D}=\int_{0}^{\varepsilon} F O_{W D} d \varepsilon
$$

During the mixing process, the stressed parts of the mixer (e.g., the fuselage, paddle, and paddle shaft), and the mixing cylinder suffer from a slight elastic deformation $\sigma$ under stress $F O_{B}$. Then, $W_{E D}$ can be calculated by:

$$
W_{E D}=\frac{1}{2} F O_{B} \sigma
$$

Combining the total stiffness of the mechanical mixer $S P=F O_{B} / \sigma$ with formula (6):

$$
W_{E D}=\frac{F O_{B}}{2 S P}
$$

When the fluid flow overcomes the frictions brought by the stressed parts or the mixing cylinder, the friction energy is mainly consumed at the contact between the paddle and the inner wall of the mixing cylinder. There is also a small friction loss at the paddle shaft, which is so small as to be negligible. Let $\varepsilon$ be the angular displacement of paddle shaft rotation per unit time. Then, the corresponding rotation angle $\omega$ can be expressed as:

$$
d \omega=\frac{2 \pi}{g} d \varepsilon
$$

When the cylinder contains no liquid to be mixed, the rotation angle of the paddle per unit time is denoted as $\beta$, the angular change brought by friction, which depends on the friction coefficient between liquid and paddle shaft/cylinder wall, is denoted as $\tau$, and the paddle diameter is denoted as $M D_{S C}$. In the paddle, the torque caused by friction can be calculated by:

$$
T O_{1}=\frac{F O_{B} M D_{S C}}{2}[\operatorname{tg}(\beta+\tau)-\operatorname{tg} \beta]
$$

Let $F R_{C}$ be the friction coefficient between the paddle and paddle shaft; $O D_{S L}$ and $I D_{S L}$ be the outer diameter and inner diameter of the slider, respectively. Then, the friction torque at the contact between the main screw and the slider can be expressed as:

$$
T O_{2}=\frac{1}{3} F R_{C} F O_{B}\left(\frac{O D_{S L}{ }^{3}-I D_{S L}{ }^{3}}{O D_{S L}{ }^{3}-I D_{S L}{ }^{3}}\right)
$$

To sum up, the total friction energy consumption of the system can be calculated by:

$$
\begin{aligned}
& W_{F R}=\int\left(T O_{1}+T O_{2}\right) d \omega \\
& =\left\{\begin{array}{l}
\frac{\pi M D_{S C}}{g}[t g(\beta+\tau)-t g \beta] \\
+\frac{2 \pi}{3 g} F R_{C}\left(\frac{O D_{S L}{ }^{3}-I D_{S L}{ }^{3}}{O D_{S L}{ }^{2}-I D_{S L}{ }^{2}}\right)
\end{array}\right\} \int F O_{B} d \varepsilon
\end{aligned}
$$

Whereas,

$$
\begin{aligned}
& \operatorname{tg} \beta=\frac{g}{\pi M D_{S C}}, \xi_{\beta+\tau}=\frac{\operatorname{tg} \beta}{\operatorname{tg}(\beta+\tau)}, \\
& \int F O_{B} d \varepsilon=\int F O_{B}\left(d \varepsilon_{E D}+d \varepsilon_{W D}\right)=W_{E D}+W_{W D},
\end{aligned}
$$

the total friction energy consumption of the system can be rewritten as:

$$
\begin{aligned}
& W_{F R}=\left[\frac{1}{\xi_{\beta+\tau}}-1+\frac{2 \pi}{3 g} F R_{C}\left(\frac{O D_{S L}{ }^{3}-I D_{S L}{ }^{3}}{O D_{S L}{ }^{2}-I D_{S L}{ }^{2}}\right)\right] \\
& \left(W_{E D}+W_{W D}\right)
\end{aligned}
$$

Combining formula (12) with formula (4):

$$
S E=\left[\frac{1}{\xi_{\beta+\tau}}+\frac{2 \pi}{3 g} F R_{C}\left(\frac{O D_{S L}{ }^{3}-I D_{S L}{ }^{3}}{O D_{S L}{ }^{3}-I D_{S L}{ }^{2}}\right)\right]\left(W_{E D}+W_{W D}\right)
$$

Let the former term in formula (13) be the kinetic energy reduction coefficient $\gamma$. Then, we have:

$$
\gamma=\frac{1}{\frac{1}{\xi_{\beta+\tau}}+\frac{2 \pi}{3 g} F R_{C}\left(\frac{O D_{S L}{ }^{3}-I D_{S L}{ }^{3}}{O D_{S L}{ }^{2}-I D_{S L}{ }^{2}}\right)}
$$




$$
W_{F R}=(1-\gamma) S E
$$

$$
W_{E D}+W_{W D}=\gamma S E
$$

That is, $W_{W D}=\gamma S E-W_{E D}$. Formula (14) shows the $\gamma$ value only relates to the structural parameters of the paddle and paddle shaft, as well as the lubrication of cylinder wall. The value is fixed for a specific mixer. Combining formulas (15) and (13):

Hence, friction work only depends on $S E$ and $\gamma$. Suppose paddle and paddle shaft have the same friction coefficient. Formula (14) can be simplified as:

$$
\gamma=\frac{\operatorname{tg} \beta}{\operatorname{tg}(\beta+\tau)+\frac{4}{5} \operatorname{tg} \tau}
$$
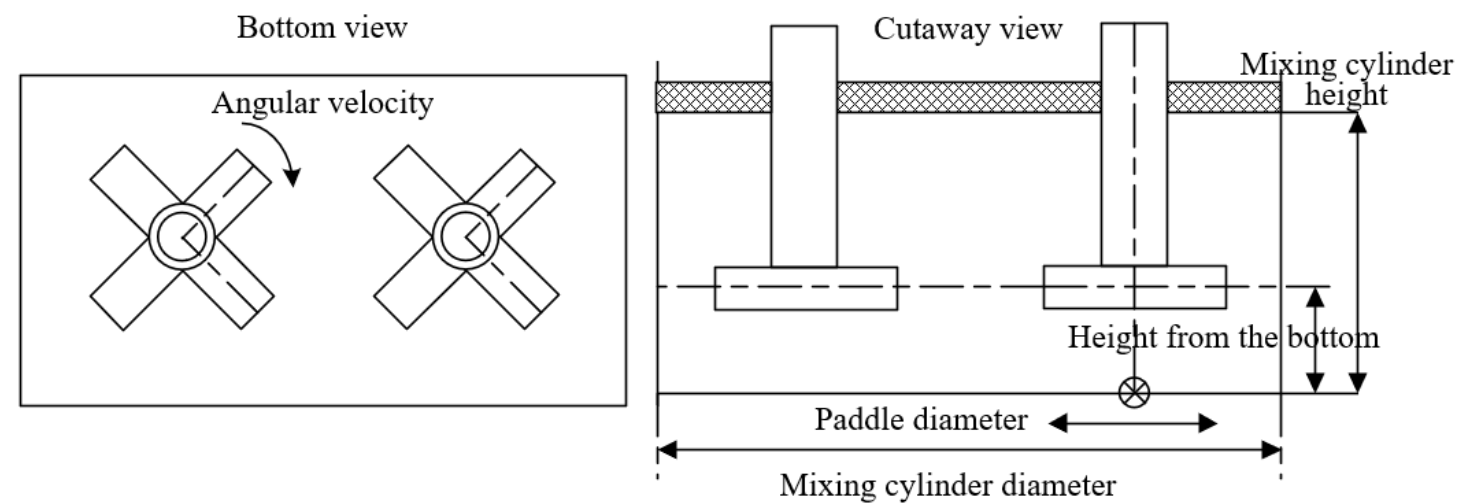

Figure 3. Geometric structure, bottom view, and cutaway view of mixing cylinder

Figure 3 shows the geometric structure, bottom view, and cutaway view of a mixing cylinder with two paddles. The mixer can operate with one or both paddles. Let $\delta$ and $P_{T C}$ be the yield stress and volume of the liquid, respectively; $B D_{0}$ and $B H_{0}$ be the liquid diameter and height in single-paddle operation, respectively; $B D_{1}$ and $B H_{1}$ be the liquid diameter and height in two-paddle operation, respectively. Then, the work $W_{W D}$ that promotes the liquid to flow in the mixing cylinder can be calculated by:

$$
W_{W D}=\delta P_{T C}\left[\ln \frac{B D_{0}}{B H_{1}}+\frac{1}{9}\left(\frac{B D_{1}}{B H_{1}}-\frac{B D_{0}}{B H_{0}}\right)\right]
$$

Suppose the effective mixing energy $S E_{0}$ equals $\gamma S E$. Then, the following can be derived from formula (15):

$$
S E_{0}=W_{W D}+\frac{F O_{B}^{2}}{2 S P}
$$

Formula (19) shows that the mixing force can be controlled, as long as the mixing energy $S E_{0}$ of the mechanical mixer is controllable. If the sample liquid has been fully mixed, the mixing force can be calculated by:

$$
F O_{B}=\sqrt{2 S P\left(S E_{0}-W_{W D}\right)}
$$

Let $M_{0}$ be the synchronous velocity of the driving motor; $M_{S}$ and $\theta_{S}$ be the velocity and angular velocity of the motor, respectively; $\eta$ be the slip ratio; $V L$ be the number of pole pairs; $F R_{S}$ be the power frequency of the motor. Since the angular velocity $\theta_{0}$ of the motor in the mechanical mixer with variable frequency control is related to its frequency, we have:

$$
M_{S}=M_{0}(1-\eta)=\frac{60 F R_{S}}{V L}
$$

$$
\theta_{S}=\frac{\pi M_{S}}{30}
$$

Substituting formulas (21) and (22) into formula (3):

$$
S E=\frac{2}{r^{2}}\left(\frac{g^{2}}{4 \pi^{2}} Q_{M}+I N\right)\left(\frac{\pi}{V L}\right)^{2}(1-\eta)^{2} F R_{S}^{2}
$$

Formula (23) can be rewritten as:

$$
S E=C_{S E}(1-\eta)^{2} F R_{S}^{2}
$$

where, $C_{S E}$ is the energy coefficient:

$$
C_{S E}=\frac{2}{r^{2}}\left(\frac{g^{2}}{4 \pi^{2}} Q_{M}+I N\right)\left(\frac{\pi}{V L}\right)^{2}
$$

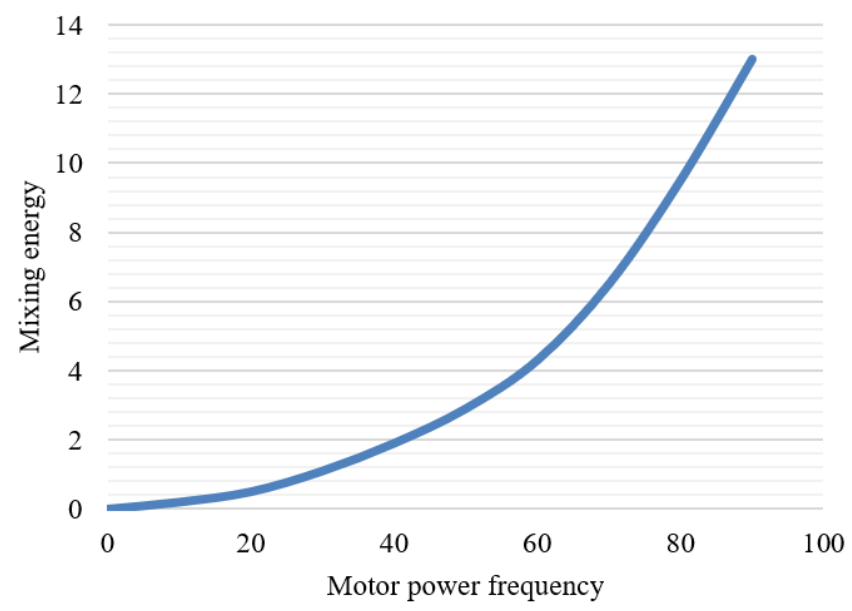

Figure 4. Relationship between mixing energy and motor power frequency 
Formula (25) shows that $C_{S E}$ is merely associated with $Q_{M}$, $I N, g, r$, and $V L$. From formula (24), it can be inferred that the mixing energy is proportional to the square of the input frequency of the inverter-controlled motor. However, the mixing velocity cannot be controlled accurately, under the influence of $\eta$. Figure 4 shows the relationship between the mixing energy and the input frequency of the invertercontrolled motor, when the $\eta$ value does not change much.

Combining formulas (24) and (20), the relationship between the mixing force and stiffness of the mechanical mixer and the frequency of the drive motor can be sorted out as:

$$
F O_{B}=\sqrt{2 S P\left[\gamma C_{S E}(1-\eta)^{2} F R_{S}{ }^{2}-W_{W D}\right]}
$$

Formula (26) shows that, for the mechanical mixer with digital variable frequency control, the accurate application of mixing force requires careful analysis on the total stiffness $S P$ of the mixer, in addition to the variable frequency control of energy. The total stiffness cannot be directly measured through experiments, but through finite-element simulation.

\section{NUMERICAL SIMULATION OF FLUID FLOW FEATURES}

This paper focuses on the flow features of the liquid in the mixing cylinder. In real-world working conditions, it is necessary to mix the liquid containing solid particles. Therefore, the liquid in the mixing cylinder needs to be modeled mathematically based on the mixture model and Euler model. The mixture model can be obtained by simplifying the Euler model, under the assumption of minimum Stokes number. But the accuracy of the model is severely limited. To overcome the limitation, this paper adopts the Euler-Euler two fluid model to numerically simulate the solid-liquid two-phase flow in mixing cylinder, which contains both solid particles and liquid.

\subsection{Governing equation}

It is assumed that there exist continuous liquid and solid phases. Let $\lambda_{i}, \tau_{i}$, and $v_{i}$ be the volume fraction, density, and velocity of phase $i$, which can characterize the liquid phase $k$ and the solid phase $u$ simultaneously. For phase $i$, the continuity equation can be constructed as:

$$
\frac{\partial}{\partial h}\left(\lambda_{i} \tau_{i}\right)+\nabla \cdot\left(\lambda_{i} \tau_{i} v_{i}\right)=0
$$

Let $a$ be the acceleration of gravity; $E F_{k}$ and $L I_{k}$ be the mixing force and liquid level lift, respectively; $V Q_{k}$ be the virtual mass force $V Q_{k}$. According to the Navier-Stokes equation, the momentum conservation equation of the liquid phase can be constructed as:

$$
\begin{aligned}
& \frac{\partial}{\partial h}\left(\lambda_{k} \tau_{k} v_{k}\right)+\nabla \cdot\left(\lambda_{k} \tau_{k} v_{k} v_{k}\right) \\
& =\sum_{u=1}^{M}\left(M E F_{u k}\left(v_{u}-v_{k}\right)+\stackrel{\square}{\left.Q_{M-u k} v_{u k}-Q_{M-u k} v_{k u}\right)}\right. \\
& -\lambda_{k} \nabla V L+\nabla \cdot L P V_{k}+\lambda_{k} \tau_{k} a+\left(E F_{k}+L I_{k}+V Q_{k}\right)
\end{aligned}
$$

Let $C V_{k}$ be the shear viscosity and $\varepsilon_{k}$ be the bulk viscosity of liquid phase $k ; M E F_{u k}$ be the solid-liquid momentum exchange coefficient; THM be the thermodynamic pressure; $v_{u k}$ be the relative velocity between solid and liquid phases. Then, the liquid phase viscous stress $L P V_{k}$ can be calculated by:

$$
\stackrel{\stackrel{=}{P} V_{k}}{=}=\lambda_{k}\left(\varepsilon_{k}-\frac{2}{3} C V_{k}\right) \nabla \cdot v_{k} \bar{J}+\lambda_{k} C V_{k}\left(\nabla v_{k}+\nabla v_{k}^{T}\right)
$$

Assuming that the momentum exchange coefficients between the solid and the liquid phases are equal $C_{k u}=C_{u k}$, then the momentum conservation equation of the solid phase can be established as:

$$
\begin{aligned}
& \frac{\partial}{\partial h}\left(\lambda_{u} \tau_{u} v_{u}\right)+\nabla \cdot\left(\lambda_{u} \tau_{u} v_{u} v_{u}\right) \\
& =-\lambda_{u} \nabla T H M-\nabla T H M_{u}+\nabla \cdot L P V_{u}+\lambda_{u} \tau_{u} a \\
& +\left(E F_{u}+L I_{u}+V Q_{u}\right) \\
& +\sum_{k=1}^{N}\left(M E F_{k u}\left(v_{k}-v_{u}\right)+Q_{k u t} v_{k u}-Q_{M-u k} v_{u k}\right)
\end{aligned}
$$

The features of the fluid in the mixing cylinder can be obtained by solving the above equations. Let $P C R_{u u}$ be the collision recovery coefficient of solid particles and liquid; $\Psi u$ be the temperature of solid particles. Then, the kinetic energy $\mathrm{THM}_{u}$ generated by the collision between solid particles can be calculated as:

$$
T H M_{u}=\lambda_{u} \tau_{u} \Psi_{u}+2 \tau_{u}\left(1+P C R_{u u}\right) \lambda_{u}^{2} a_{0, u u} \Psi_{u}
$$

where, $a_{0, u u}$ is the radial distribution function:

$$
a_{0, u u}=\left[1-\left(\frac{\lambda_{u}}{\lambda_{u, \max }}\right)^{1 / 3}\right]^{-1}
$$

The translation and collision of the solid particles cause their momentum to be exchanged. During the exchange, the resulting shear viscosity $C V_{u}$ and volume viscosity $V O_{u}$ constitute the stress tensor $L P V_{u}$ of the solid particles. Let $C V_{u-}$ cla, $C V_{u-d y}$, and $C V_{u-r u b}$ be the collision viscosity, dynamic viscosity, and friction viscosity of solid particles, respectively. Then, $C V_{u}$ can be expressed as:

$$
C V_{u}=C V_{u-c l a}+C V_{u-d y}+C V_{u-r u b}
$$

Then, $C V_{u-c l a}$ and $C V_{u-d y}$ can be respectively modeled as:

$$
\begin{gathered}
C V_{u-c l a}=\frac{4}{5} \lambda_{u} \tau_{u} H D_{u} a_{0, u u}\left(1+P C R_{u u}\right)\left(\frac{\psi_{u}}{\pi}\right)^{1 / 2} \lambda_{u} \\
C V_{u-d y}=\frac{\lambda_{u} H D_{u} \tau_{u} \sqrt{\psi_{u} \pi}}{6\left(3-P C R_{u u}\right)} \\
{\left[1+\frac{2}{5}\left(1+P C R_{u u}\right)\left(3 P C R_{u u}-1\right) \lambda_{u} a_{0-u u}\right]}
\end{gathered}
$$


Let $v$ be the internal friction angle; $J_{2 T}$ be the second invariant of the deviatoric stress tensor. Then, $C V_{u-r u b}$ can be modeled as:

$$
C V_{u-r u b}=\frac{T H M_{u} \sin v}{2 \sqrt{J_{2 T}}}
$$

The resistance force $V O_{u}$ of solid particles against the liquid-induced compression and expansion can be expressed as:

$$
\varepsilon_{u}=\frac{4}{3} \lambda_{u}^{2} \tau_{u} H D_{u} a_{0, u u}\left(1+P C R_{u u}\right)\left(\frac{\psi_{u}}{\pi}\right)^{1 / 2}
$$

\subsection{Drag model}

The most important dynamic feature between the two phases is their interaction. During the momentum exchange between the liquid and solid two phases, the dominant force is drag, that is, the resistance to the dispersion phase produced through the relative friction and slip between the two phases. For a dense solid-liquid system, the drag is often described by the Gidaspow model, a combination between Wen-Yu model and Ergun model:

$$
\begin{aligned}
& M E F_{u k}=\frac{3}{4} S P_{T} \frac{\lambda_{u} \lambda_{k} \tau_{k}\left|v_{u}-v_{k}\right|}{H D_{u}} \lambda_{k}^{-2.65} \\
& \frac{\partial}{\partial h}\left(\tau_{k} b_{k} p\right)+\nabla\left(\tau_{k} b_{k} v_{k} p\right)=\nabla\left(b_{k} \frac{C V_{k}}{c_{l}} \cdot \nabla p\right)+b_{k} C V_{k}\left[\nabla v_{k}+\nabla\left(v_{k}\right)^{T}\right]: \nabla v_{k}-\tau_{k} b_{k} b \\
& \frac{\partial}{\partial h}\left(\tau_{k} b_{k} b\right)+\nabla\left(\tau_{k} b_{k} v_{k} b\right)=\nabla\left(\lambda_{k} \frac{C V_{k}}{c_{\lambda}} \cdot \nabla b\right)+C O V_{1} b_{k} C V_{k}\left\{\left[\nabla v_{k}+\nabla\left(v_{k}\right)^{T}\right]: \nabla v_{k}\right\} \frac{b}{l}-\tau_{k} b b_{k} b \frac{b^{2}}{l}
\end{aligned}
$$

$$
S P_{T}=\frac{24}{\lambda_{k} E W_{u}}\left[1+0.15\left(\lambda_{k} E W_{u}\right)^{0.687}\right]
$$

Considering the density of solid-liquid two phase in the system, the drag coefficient needs to be modified to increase calculation accuracy. Let $\Gamma$ be the Kolmogorov length. Then, the Brucato modified model can be adopted:

$$
S P_{T 0}=S P_{T}\left[1+8.76 \times 10^{-4}\left(\frac{H D_{T H M}}{\Gamma}\right)^{3}\right]
$$

where, $L M V$ is the kinematic viscosity of the liquid phase:

$$
\Gamma=\left(\frac{L M V^{3}}{\lambda}\right)^{0.25}
$$

\subsection{Turbulence model}

Ignoring the influence of molecular viscosity and dispersed particles on the liquid phase, this paper constructs a complete turbulence model for the liquid in the mixing cylinder based on the standard $l-\lambda$ model. The turbulence length and time scale were determined by solving two independent transport equations. Let $p$ and $b$ be the kinetic energy and dissipation rate of the turbulence, respectively. Under the constants of $C O V_{1}=1.45, C O V_{2}=1.95, c_{l}=1.0$, and $c_{\lambda}=1.0$, the turbulent kinetic energy transport equations can be established as:
Let $C O V_{C V}$ be the turbulence model constant. Then, the turbulent viscosity coefficient $C V_{k}$ of the liquid phase can be calculated by:

$$
C V_{k}=C O V_{C V} \tau \frac{p^{2}}{b}
$$

Here, the paddle interval is $1.25 \mathrm{~m}$, the distance of paddle to cylinder bottom is $0.275 \mathrm{~m}$, and the coordinates of the two paddles are $(-0.625,0,0.275)$ and $(0.625,0,0.275)$, respectively.

\section{EXPERIMENTS AND RESULTS ANALYSIS}

Figure 5 presents the dimensions of the internal structure of the mixing cylinder in the mechanical mixer for our experiments. The shaft distance between the two paddles is $1.4 \mathrm{~m}$; the distance of paddle to cylinder bottom is $0.25 \mathrm{~m}$, and the center coordinates of the two paddles are $(-0.7,0,0.25)$ and $(0.7,0,0.25)$, respectively.

Figures 6 (a) and (b) present the variation in velocity vectors of liquid flow under single and two paddle operations, respectively. Under both operation modes, each paddle mixed the liquid and solid particles below under the drive of the motor. Due to the helical diffusion effect, the vortex created by the paddle near the bottom of the mixing cylinder gradually widened; the bending radius of the trailing vortex induced by interference gradually extended; a backflow was formed between the blades, and each blade and the mixing cylinder wall.

Figure 7 presents the steady-state residual curve of the liquid flow field during the initial mixing phase of the test system, which verifies that the good stability of the initial mixing flow field.

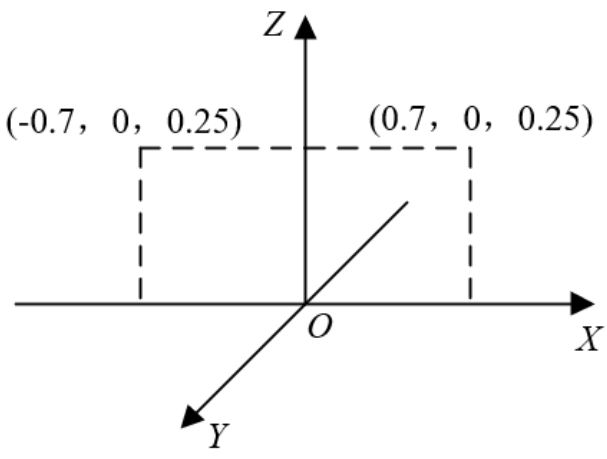

Figure 5. Dimensions of the internal structure of the mixing cylinder 


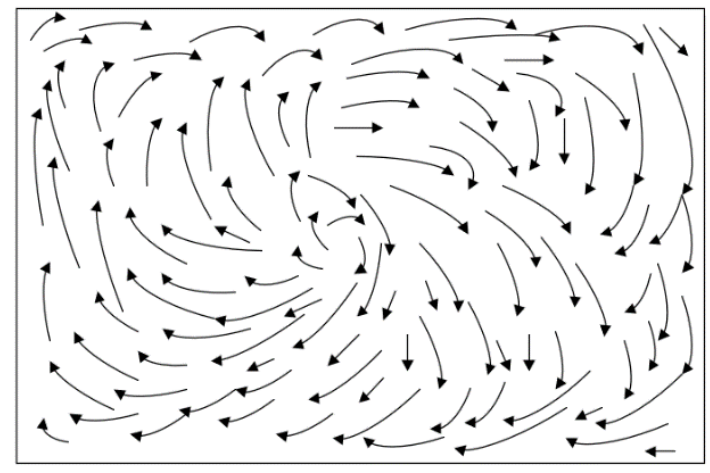

(a) Single paddle operation

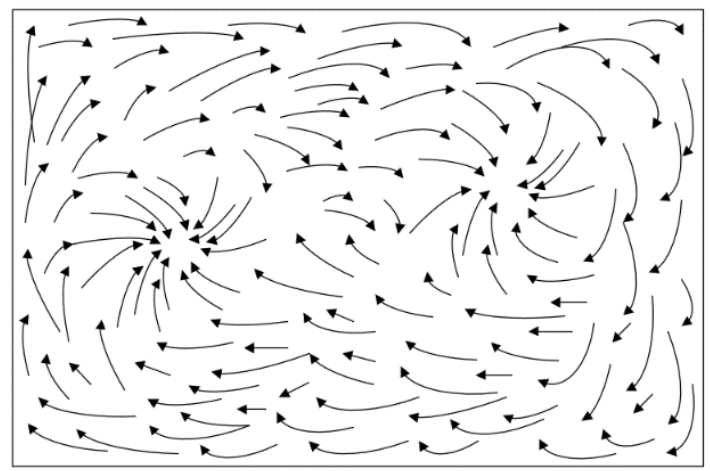

(b) Two paddle operation

Figure 6. Velocity vectors of liquid flow under single and two paddle operations

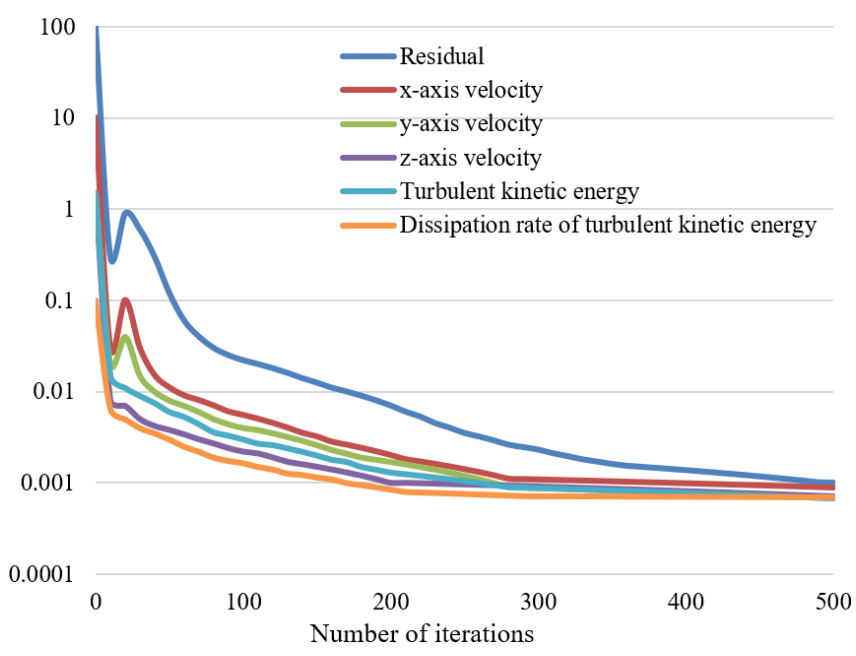

Figure 7. Initial steady-state residual curve

Figures 8 (a) and (b) show the changes in mean radial velocity and mean axial velocity of the liquid phase at different particle volume fractions, respectively. As shown in Figure $8(\mathrm{a})$, the peak of the mean radial velocity at the paddle end

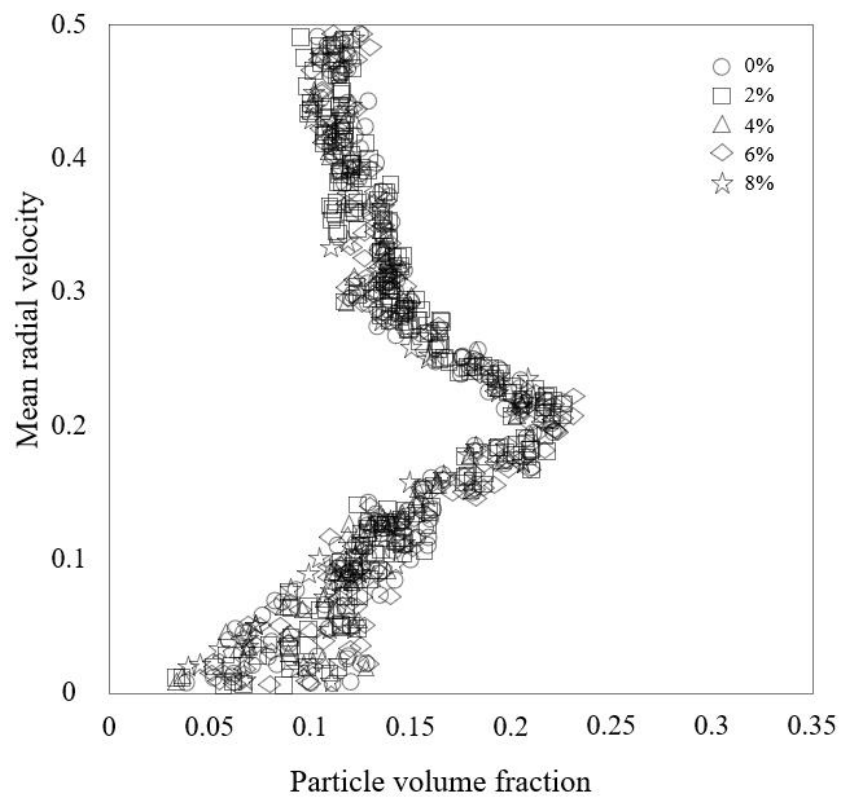

(a) Mean radial velocity gradually decreased with the growing particle volume fraction, and the decrement was the largest as the latter increased from 0.01 to 0.25 . At the bottom of the mixing cylinder, the mean radial velocity of the liquid phase exhibited the same trend as that in the other regions. However, the velocity change lagged in this region, because the fluid flowed slowly in the presence of solid particles. As shown in Figure 8(b), the mean axial velocity of the liquid phase gradually decreased with the growing particle volume fraction, and the solid phase concentration increased accordingly on the mixing cylinder wall, where the largest decrement of mean axial velocity occurred for the liquid phase.

This paper quantitatively compares the influence of particle volume fractions on radial and axial pulsation velocities. The variations in the two velocities are recorded in Figure 9. As shown in Figure 9(a), the turbulent pulsation of the liquid phase was weakened under the influence of solid particles. As the particle volume fraction changed from 0.01 to 0.25 , the radial pulsation velocity of the liquid phase plunged clearly by an average of $18 \%$. As shown in Figure 9(b), as the particle volume fraction changed from 0.01 to 0.26 , the axial pulsation velocity of the liquid phase dropped by an average of $16 \%$.

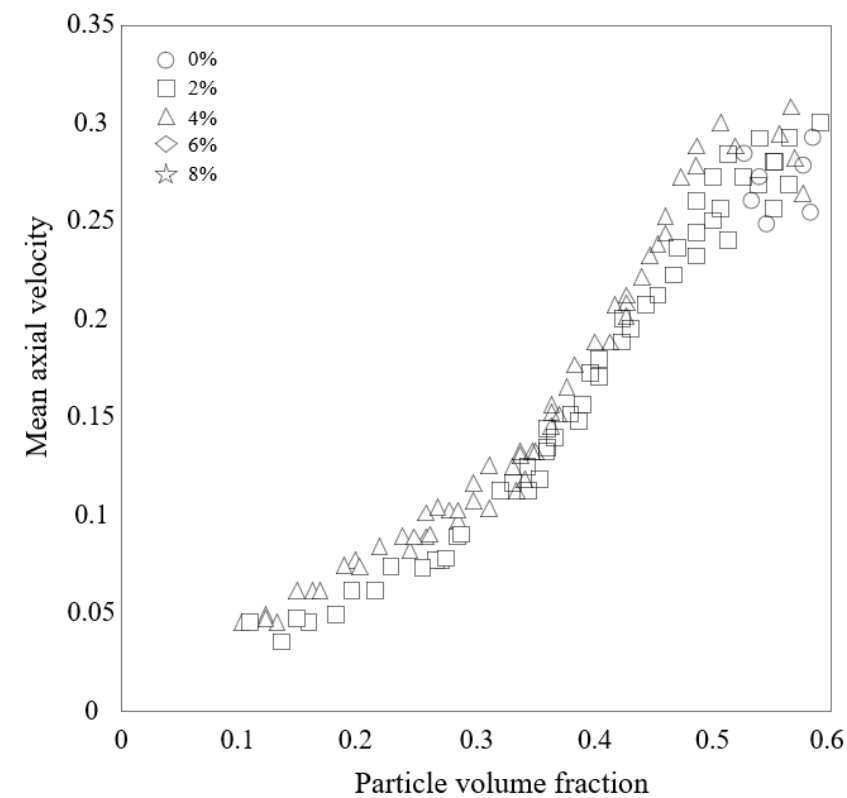

(b) Mean axial velocity

Figure 8. Variation in mean radial and axial velocities at different particle volume fractions 


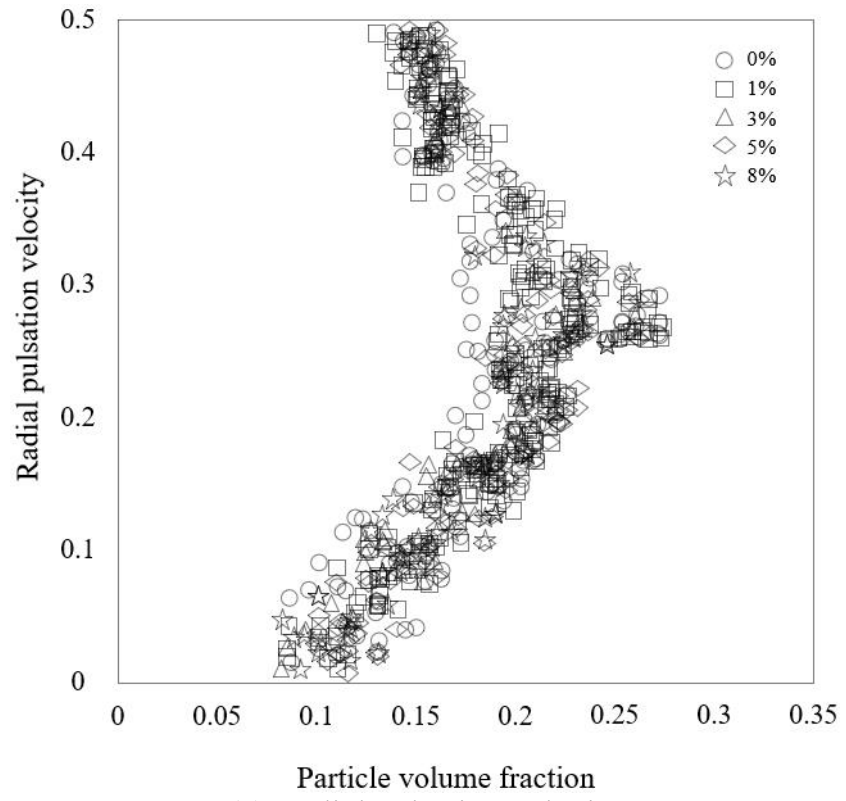

(a) Radial pulsation velocity

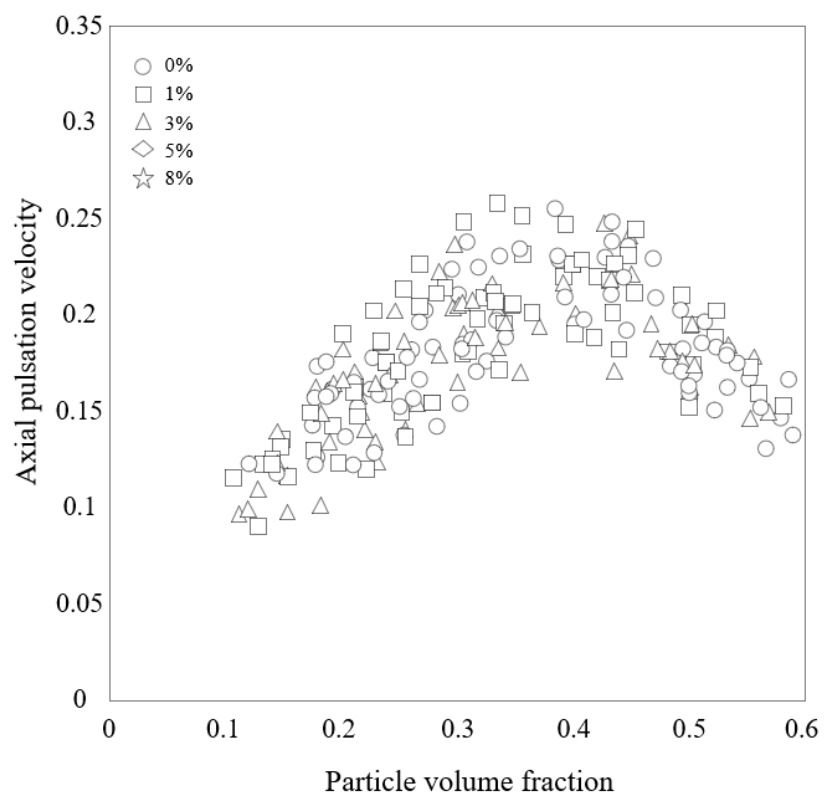

(b) Axial pulsation velocity

Figure 9. Variation in mean radial and axial pulsation velocities at different particle volume fractions

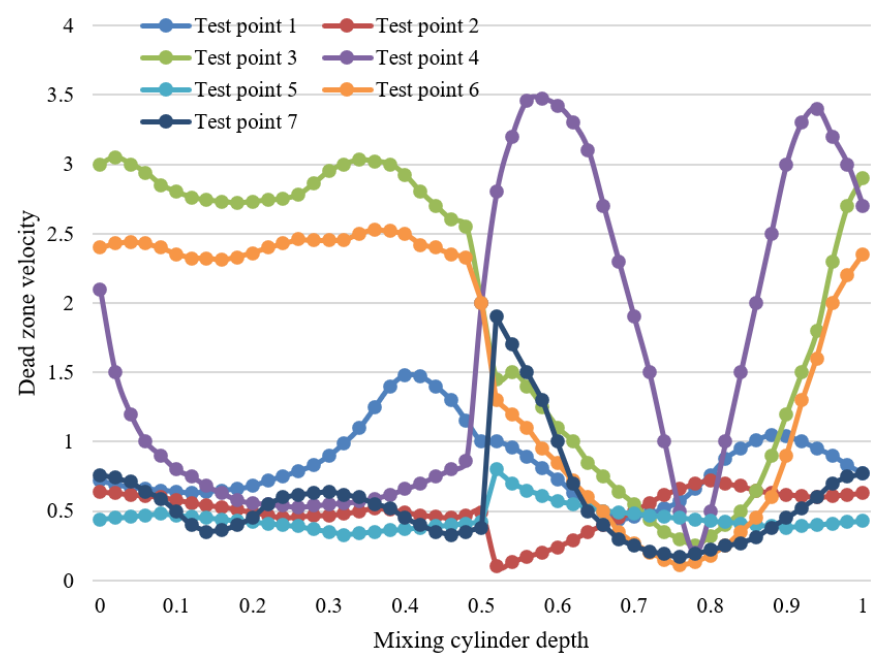

Figure 10. Variation in dead zone velocity with mixing cylinder depths

Figure 10 records the variation in dead zone velocity with mixing cylinder depths. In the mixing cylinder, a dead zone of solid particle mixing appeared with the velocity of less than $0.35 \mathrm{~m} / \mathrm{s}$ within the depth range of $0.1-0.38 \mathrm{~m}$ in the mixing cylinder. The observation verifies that the low-velocity zone in the axial direction is not greatly disturbed by the paddle action. This dead zone causes some solid particles to deposit and fall to the bottom of the cylinder, making it hard for them to collide and merge with liquid.

\section{CONCLUSIONS}

This paper carries out numerical simulation of the flow features of the fluid in the mechanical mixer with automatic variable frequency control, creates a reasonable structure for the application system of the said mixer, and specifies the basic devices and number of input/output points of the system. On this basis, the relationship between the force energy and control frequency of the mixing cylinder was detailed. Next, the Euler-Euler two fluid model was introduced to numerically simulate the solid-liquid two-phase flow in the cylinder of the mixer, and the relevant models were set up for control loop, drag, and turbulence. Through experiments, the authors captured the variation in fluid flow velocity vectors and the steady-state residual curves, and verified the good stability of the mixing flow field in the initial phase. After that, comparative analyses were conducted on the mean radial and axial velocities, as well as the mean radial and axial pulsation velocities of the liquid phase under different particle volume fractions. Finally, the variation in dead zone velocity with mixing cylinder depths was tested. The experimental results fully demonstrate the effectiveness of our model, and provide a good reference for the relevant research and the optimal design of the drive module of mixers.

\section{REFERENCES}

[1] Gires, P.Y., Thampi, M., Weiss, M. (2020). Quantifying active diffusion in an agitated fluid. Physical Chemistry Chemical Physics, 22(38): 21678-21684. https://doi.org/10.1039/d0cp03629c

[2] Camacho Corzo, D.M., Ma, C.Y., Mahmud, T., Roberts, K.J. (2020). Digital design of batch cooling crystallization processes: computational fluid dynamics methodology for modeling free-surface hydrodynamics in agitated crystallizers. Organic Process Research \& Development, 24(11): 2565-2582. https://doi.org/10.1021/acs.oprd.0c00240

[3] Reviol, T., Kluck, S., Genuit, F., Reim, V., Böhle, M. (2016). Investigation of the influence of viscoelastic behaviour on the agitation of non-Newtonian fluid flow. Chemical Engineering Science, 152: 55-64. https://doi.org/10.1016/j.ces.2016.05.035

[4] Rajabi, M., Arghavani-Beydokhti, S., Barfi, B., Asghari, A. (2017). Dissolvable layered double hydroxide as an efficient nanosorbent for centrifugeless air-agitated dispersive solid-phase extraction of potentially toxic metal ions from bio-fluid samples. Analytica Chimica Acta, 957: 1-9. https://doi.org/10.1016/j.aca.2016.12.041 
[5] Jaszczur, M., Młynarczykowska, A., Demurtas, L. (2020). Effect of impeller design on power characteristics and Newtonian fluids mixing efficiency in a mechanically agitated vessel at low Reynolds Numbers. Energies, 13(3): 640. https://doi.org/10.3390/en13030640

[6] Jasikova, D., Kotek, M., Kopecky, V. (2015). Time resolved PIV measurement of fluid dynamics in agitated vessels. Proceedings of the SPIE, 9442: $94420 \mathrm{H}$. https://doi.org/10.1117/12.2176018

[7] Chamsartra, S., Hewitt, C.J., Nienow, A.W. (2005). The impact of fluid mechanical stress on Corynebacterium glutamicum during continuous cultivation in an agitated bioreactor. Biotechnology Letters, 27(10): 693-700. https://doi.org/10.1007/s10529-005-4690-5

[8] Chaalal, O. (2005). An experimental study of fluid motion in a bioreactor agitated by line-source bubble plumes. Energy Sources, 27(1-2): 61-71. https://doi.org/10.1080/00908310490448118

[9] Kiebert, F., König, J., Kykal, C., Schmidt, H. (2015). Measurements of streams agitated by fluid loaded sawdevices using a volumetric 3-component measurement technique (v3v). Physics Procedia, 70: 25-29. https://doi.org/10.1016/j.phpro.2015.08.018

[10] Hammami, M., Chebbi, A., Baccar, M. (2013). Numerical study of hydrodynamic and thermal behaviors of agitated yield-stress fluid. Mechanics \& Industry, 14(4): 305-315. https://doi.org/10.1051/meca/2013070

[11] Salek, M.M., Sattari, P., Martinuzzi, R.J. (2012). Analysis of fluid flow and wall shear stress patterns inside partially filled agitated culture well plates. Annals of Biomedical Engineering, 40(3): 707-728. https://doi.org/10.1007/s10439-011-0444-9

[12] Hosseini, S., Patel, D., Ein-Mozaffari, F., Mehrvar, M. (2010). Study of solid- liquid mixing in agitated tanks through computational fluid dynamics modeling. Industrial \& Engineering Chemistry Research, 49(9): 4426-4435. https://doi.org/10.1021/ie901130z

[13] Rotkiske, T.A., Bostater, C.R. (2016). GIS mapping of fluid mud transport pre-, during-, and post-dredging agitation, by using engineered novel instrumentation. The 26th International Ocean and Polar Engineering Conference, Rhodes, Greece, pp. 1542-1547.
[14] Panneerselvam, R., Savithri, S., Surender, G.D. (2009). Computational fluid dynamics simulation of solid suspension in a gas - liquid- solid mechanically agitated contactor. Industrial \& Engineering Chemistry Research, 48(3): 1608-1620. https://doi.org/10.1021/ie800978w

[15] Meridiano, G., Weheliye, W.H., Mazzei, L., Angeli, P. (2018). Experimental and computational studies of the fluid dynamic behaviour of liquid-solid mixtures in agitated vessels. AIChE Annual Meeting, pp. 23-26.

[16] Benmoussa, A., Rahmani, L. (2018). Numerical Analysis of thermal behavior in agitated vessel with NonNewtonian fluid. The International Journal of Multiphysics, 12(3): 209-220. tps://doi.org/10.21152/1750-9548.12.3.209

[17] Reviol, T., Kluck, S., Böhle, M. (2018). A new design method for propeller mixers agitating non-Newtonian fluid flow. Chemical Engineering Science, 190: 320-332. https://doi.org/10.1016/j.ces.2018.06.033

[18] Triveni, B., Vishwanadham, B., Madhavi, T., Venkateshwar, S. (2010). Mixing studies of nonNewtonian fluids in an anchor agitated vessel. Chemical Engineering Research and Design, 88(7): 809-818. https://doi.org/10.1016/j.cherd.2009.11.020

[19] Ricci, E., Kelly, W.J. (2004). The effects of power law fluid rheology on coil heat transfer for agitated vessel in the transitional flow regime. The Canadian Journal of Chemical Engineering, 82(6): 1275-1283. https://doi.org/10.1002/cjce.5450820615

[20] Zughbi, H.D., Rakib, M.A. (2004). Mixing in a fluid jet agitated tank: effects of jet angle and elevation and number of jets. Chemical Engineering Science, 59(4): 829-842. https://doi.org/10.1016/j.ces.2003.09.044

[21] Zughbi, H.D., Rakib, M.A. (2002). Investigations of mixing in a fluid jet agitated tank. Chemical Engineering Communications, 189(8): 1038-1056. https://doi.org/10.1080/00986440213878

[22] Wu, B., Kennedy, S., Eshtiaghi, N., Parthasarathy, R. (2018). CFD modeling of active volume creation in a non-Newtonian fluid agitated by submerged recirculating jets. Chemical Engineering \& Technology, 41(7): 1441-1447. 